\title{
Polarization Microscopy Study of Jet Fuel Crystallization
}

\section{Marlin Vangsness}

University of Dayton Research Institute, 300 College Park, Dayton, OH 45469+0116

\section{Introduction}

Long duration operation of aircraft at high altitude may subject jet fuel to freezing temperatures. Prolonged exposure to low temperatures can cause reduced fuel flowdue to increased viscosity and plugged filters. If unchecked, fuel freezing may result in the catastrophic loss of aircraft and crew. An alternative to the use of specialty fuels, which have lower freeze points, is the employment of fuel additives. In order to choose additive candidates intelligently we need to increase our understanding of how additives influence the freezing of jet fuel. Using a coldstage microscope to slowly cool the fuel allows us to examine the crystal microstructure as it forms. This information is important in the development of models used in the numerical simulation of fuel freezing within a fuel system, and with the interpretation of other fundamental low-temperature experiments.

\section{Experimental}

With conventional microscope optics and lighting, it is difficult to distinguish between solid and liquid fuel. In the temperature range of interest ( $-45 \mathrm{C}$ to $-70 \mathrm{C}$ ), the solid portion of the freezing jet fuel is believed to consist primarily of normal alkanes which. Normal alkane crystals may be orthorhombic, triclinic or in some cases monoclinic. With a different index of refraction along each axis, these crystals rotate the polarization of any light not propagating along the optical axis. Crystals illuminated by polarized light and viewed through a polarization analyzer rotated perpendicular to the polarizer appear bright or colored against a dark field or in some cases, dark against a gray field. A polarized light microscope (Olympus Bmax50) with phase contrast optics and a digital camera (Sony DKC-5000) were used to record images during the freezing process (Figure 1). Approximately $20 \mu \mathrm{L}$ of fuel was placed on a microscope cover slip ( $25 \mathrm{~mm} \times 25 \mathrm{~mm} \times 1.8 \mathrm{~mm})$ and covered with a smaller $(\mathrm{d}=12.5 \mathrm{~mm})$ cover slip. The slide was placed on the liquid nitrogen cooled cold stage (Linkam), isolated from ambient conditions by an insulated pad below, a leat shield above and purged continuously with dry nitrogen. The sample was brought to-45 $\mathrm{C}$ rapidly at a rate of $30 \mathrm{C} / \mathrm{min}$. and held for 5 minutes to allow the system to equilibrate. At $-45 \mathrm{C}$, each fuel sample is above the cloud point temperature, and is not expected to freeze. The sample was then cooled at a slower rate, (typically $0.25 \mathrm{C} / \mathrm{min}$ ) while images of fuel crystallization were recorded. The sample was illuminated through a $2 \mathrm{~mm}$ hole in the cold stage.

For this study A Jet A-1 fuel, F3219, and a proprietary low-temperature additive, F3744 at a concentration of $4000 \mathrm{mg} / \mathrm{L}$, were used. From gas chromatography it was determined that F3219 is up of roughly $20 \%$ n-alkanes in the range $C_{9}$ to $C_{16}$, centered about $C_{12}$, with the balance consisting of aromatics, cycloalkanes and branched alkanes. In Jet A-1 the crystalization behavior of the alkane components is quite variable. Both ribbon and plate structures are visible, however, the flat plate structure shows marked differences between fuels.

Figure (1) illustrates the crystal growth by subtracting an image of the fuel at a later stage in freezing from an earlier image. Light shaded areas around existing crystals indicate material added during the cooling interval. This technique illustrates that in the neat fud, Figure 1(A), both plate structures and ribbon, or dendritic, structures grow across the field as the fuel is cooled from-53.5 C to $-54 \mathrm{C}$. As freezing progresses, material is added to both types of crystal habit.Figure 1(B) 
illustrates the freezing characteristics of F3219 combined with F3744. As the mixture is cooled from $-53.6 \mathrm{C}$ to -54 the majority of initial crystal growth appears to be in the form of ribbons or dendrites with most of the new growth at the end of the ribbon.

\section{Conclusion}

Photomicrographs of freezing fuel clearly show the effect of F3744, a crystal modifier, on the crystallization process. This information allows us to evaluate the effectiveness of a particular additive without more costly long duration testing and can be used qualitatively to compare against computational models.

\section{References}

[1] This work was supported by the U.S. Air Force, Air System Command, Reconnaissance System Program Office, Program Manager Barb Wellmeier; U.S. Air Force, Warner Robins Air Logistic Command, U-2 Special Programs Office, Project Manager George "Doug" Hanlin; and the U.S. Air Force Research Laboratory, Propulsion Directorate Turbine Engine Division, Wright-Patterson Air Force Base, Ohio, under Contract No. F33615-97-C-2719 (Technical Monitor: R.W. Morris).
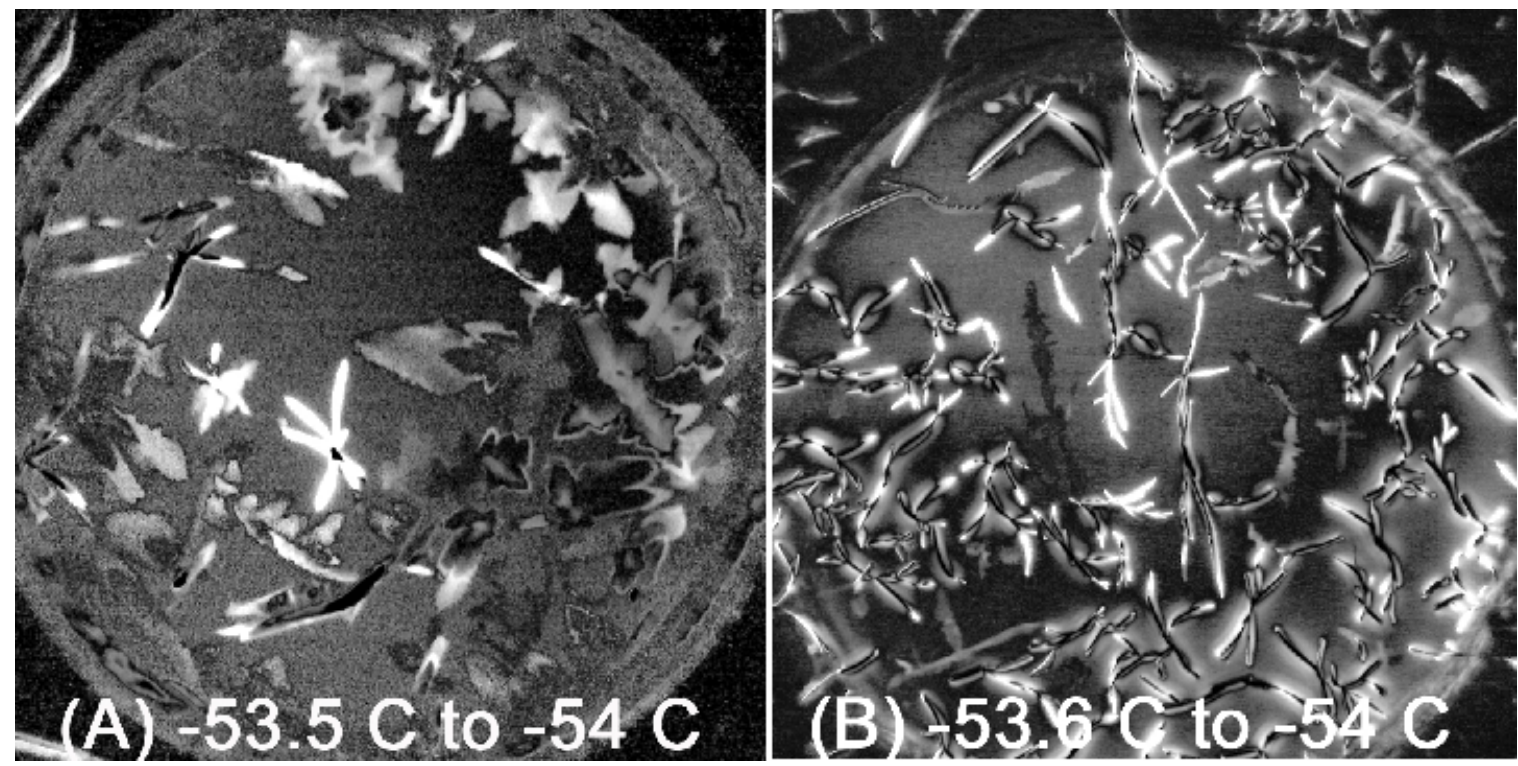

Figure 1 Crystal formation sequences. The field of view is $2 \mathrm{~mm}$. by $2 \mathrm{~mm}$. 